\title{
Über einige neue Hummelformen (Bombus), besonders aus Asien. (Hym.)
}

Von Dr. H. Friese, Schwerin i. M.

Die grofse Variabilität des farbigen Haarkleides bei den asiatischen Hummeln ist ja hinreichend bekannt, aber auch die Arten der orientalischen Region - Indien, China und JavaSumatra - scheinen hierin in nichts nachzustehen. Im folgenden führe ich die erste Serie der mir näher bekannt gewordenen Formen auf.

\section{Übersicht der Formen}

aus der Gruppe des Bombus haemorrhoidalis-orientalis Sm.

$$
\text { q } 90^{\top} \text {. }
$$

Segment 3 mehr weniger schwarz behaart

Segment 3 wie $4-5$ rot behaart

$$
\text { Gruppe B. orientalis Sm. }
$$

Gruppe B. haemorrhoidalis Sm.

\section{Bombus orientalis Sm.}

1. Segment 1-2 gelblich behaart . . . . . . . 2 . Segment 1 gelb behaart, Wangen lang B. magrettii Grib.

2. Wangen $1 \frac{1}{2}$ mal so lang wie am Ende breit. . . . 3 . Wangen viel kürzer als am Ende breit . . . . . 4.

3. Thoraxseiten weifslich behaart . . var. pectoralis n. var. Thoraxseiten schwarz behaart . . . B. orientalis Sm.

4. Thoraxseiten schwarz behaart, Mandibel gezähnt

$$
\text { B. dentatus Handl. }
$$

Thoraxseiten gelblich behaart, Mandibel ?

B. channicus Grib.

Bombus haemorrhoidalis Sm.

1. Kopf verlängert, Wangen $1^{1} / 2$ mal so lang wie breit . 2 . Kopf kurz, Wangen kürzer als am Ende breit . . . 3.

2. Thoraxseiten schwarz behaart $B$. haemorrhoidalis Sm. $q \sigma^{\top}$. Thoraxseiten weifs behaart $B$. v. albopleuralis n. var. $q$.

3. Thoraxseiten schwarz behaart, Wangen kaum $1 / 2$ so lang wie am Ende breit, Mandibel gezähnt $B$. orichalceus n. sp. $q$.

Thoraxseiten weifs behaart, Wangen $2 / 3$ so lang wie am Ende breit, Mandibel ganzrandig. B. rotundiceps n. sp. $q \sigma^{\top}$.

$$
\text { Bombus orichalceus n. sp. 우. }
$$

1880. B. haemorrhoidalis Morawitz, † in: Mélang. biolog. v. 10 p. 453 (nec Smith). 
Wie B. haemorrhoidalis Smith $q$ gefärbt, aber Kopf kurz, Wangen kaum $1 / 2$ so lang wie am Ende breit, Mandibel lang gezähnt; Thorax ganz schwarz behaart, Segment 1-3 gelb, Seiten von Segment 3 und $4-6$ rot behaart. Ventralsegment 1-2 schwarz, 3-6 rötlich gefranst. Beine fast schwarz, Tarsen rotbraun, schwarz behaart. Flügel blauschwarz. L. $22 \mathrm{~mm}, \mathrm{Br} .8 \mathrm{~mm}$.

1 ㅇ von Simla, im April 1897, Nurse leg.

Bombus orientalis var. pectoralis n. var. +9 ○.

Wie $B$. orientalis Smith (1854), aber Thoraxseiten weifs behaart, beim $\sigma^{\top}$ oft weniger reichlich vorhanden; Segment 1-2 schön und reichlich gelb behaart.

․ L. $22 \mathrm{~mm}$, Br. $8^{1 / 2} \mathrm{~mm}$, von Khasia Hills (Sla de $\mathrm{n}$ ).

․ L. $14 \mathrm{~mm}, \mathrm{Br} .5 \mathrm{~mm}$.

o. L. $17 \mathrm{~mm}$, Br. $7 \mathrm{~mm}$. 9 und $\sigma^{7}$ mehrfach von British Bootan, Padong, Durell leg. 1913, ơ auch vom Himalaja.

Bombus funerarius var. lateritius n. var. ㅇ.

ㅇ. Wie $B$. funerarius Smith (1852), aber Segment 4-6 rot behaart, Thorax und Segment 1 dagegen silbergrau behaart, $2-3$ schwarz. L. $22 \mathrm{~mm}, \mathrm{Br} .8 \mathrm{~mm}$.

4 ㅇ von Sikkim und Darjeeling im Himalaja.

Bombus haemorrhoidalis var. albopleuralis n. var. 우.

Wie B. haemorrhoidalis Sm. (1853), aber kleiner und Thoraxseiten weifs behaart; Clypeus und Beine zeigen ebenfalls die charakteristische rotbraune Färbung mit der schwarzen Behaarung des $B$. haemorrhoidalis. ㅇ. L. $18 \mathrm{~mm}$, Br. $7^{1 / 2} \mathrm{~mm}$. ․ L. $14 \mathrm{~mm}$, Br. $4^{1} / 2 \mathrm{~mm}$.

우 von Kumaon im Himalaja-Geb. am 21. Juni 1910.

B. haemorrhoidalis Sm. q mifst in der Länge $23-25 \mathrm{~mm}$, in der Breite 9-10 mm, P L. $15 \mathrm{~mm}$, Br. $5^{1 / 2} \mathrm{~mm}$; ich erhielt die Exemplare (q) von Sikkim und Dshelumm, am 18. Mai 1912 fliegend, ( $($ ) von Sikkim und Kasauh Niw P. im September gefangen.

$$
\text { Bombus rotundiceps n. sp. 웅. }
$$

Wie $B$. haemorrhoidalis var. albopleuralis gefärbt, aber fast noch kleiner, Wangen kurz, ca. ${ }^{2} / 3$ so lang wie am Ende breit; Mandibel ganzrandig, Thoraxseiten und die Seiten der hinteren Thoraxwand weifs behaart, Segment 1-2 und Scheibe von Segment 3 goldgelb behaart, Ende rot behaart. Beine rotbraun, schwarz behaart.

ㅇ. L. $16 \mathrm{~mm}, \mathrm{Br} .7 \mathrm{~mm}$. 
$\sigma^{7}$ wie \&, aber auch der Kopf, Pronotum und Femur I und II reichlich weifs behaart. $\sigma^{7}$. L. $13 \mathrm{~mm}, \mathrm{Br} .4^{1 / 2} \mathrm{~mm}$.

o von Dehsa Dun, 13. April, ơ von Kumaon im HimalajaGeb., am 9. Mai.

Bombus latissimus Friese 9.

1910. B. l. Friese, $q$ in: Verh. zool. bot. Ges. Wien p. 405 .

9. Wie das \&, nur Thorax fast schwarz behaart. L. $18 \mathrm{~mm}$, Br. $9 \mathrm{~mm}$.

19 von Formosa im März fliegend.

Bombus latissimus var. detritus n. var. P.

Wie B. latissimus, aber Kopf, Thorax und Segment 1-2 lang und dicht schwarz behaart, Segment 3-6 rot behaart. L. 12 bis $18 \mathrm{~mm}$, Br. 5-71/2 $\mathrm{mm}$ (also in sehr verschiedenen Grölsen auftretend).

9 mehrfach von Formosa bei Hoozan im November, Dezember und Januar (1909/10) und bei Taihorin im März, April und Mai, S a u ter leg.

Bombus latissimus var. tricoloratus n. var. 9.

Wie B. l. var. detritus, aber Segment 1 lang gelblichrot behaart, 2 schwarz (oft auch noch die Basis von 3), 3-6 rot behaart. L. $14-17 \mathrm{~mm}, \mathrm{Br} .6^{1} / 2-8 \mathrm{~mm}$.

$\varphi$ mehrfach von Formosa bei Taihorin im März und April fliegend, S a u te r leg.

Bombus latissimus var. segmentarius n. var. 9.

Wie $B$. $l$. var. detritus, aber Segment $1-3$ lang schwarz behaart, 3-6 schwarz. L. $11-20 \mathrm{~mm}, \mathrm{Br} .5-8 \mathrm{~mm}$.

9 mehrfach von Formosa bei Hoozan im November, Dezember und Januar und bei Taihorin im April und März fliegend.

Bombus hortorum var. castaneus n. var. 우.

․ Wie $B$. $h$. var. fuliginosus Fr., aber Beine schwarz; Kopf und Thorax schwarz behaart, Scutellum rötlichbraun, Segment 1-3 kastanienbraun, Basis von Segment 2 und 3 mit mehr weniger schwarzen Haaren.

2 으 vardinien (Assuni), 1910.

Bombus hortorum vä. basizonus n. var. +9.

ๆ. Wie die Bombus-Form argillaceus Scop., aber Segment 1 mit mehr weniger gelben Haaren oder gelber Binde am Endrande und Segment 4 jederseits am Seitenrande mit weifsem Haarfleck.

o mehrfach vom Kaukasus (Murut). 
Bombus hortorum var. trizonatus n. sp. +.

․ Wie die Bombus-Form argillaceus, aber Segment 1 mit gelben Haaren besetzt.

$q$ von Bozen (S.-Tirol), Anfang Mai unter der Stammform auf Lamium album.

Bombus hortorum var. turcestanicus n. var. ㅇ.

․ Wie B. fedtschenkoi Mor., aber nur Segment 1 gelbbraun behaart und die Basis von Segment 5 weifs behaart.

우 von Turkestan, 1898.

Bombus hortorum var. mongolicus n. var. ㅇ.

9. Wie B. ussurensis Rad., aber Segment 1-4 überall gelbbraun behaart, Segment 5 nur an der Basis und am Endrand gelbbraun behaart, also mit schwarzer Scheibenbinde, 6 schwarz behaart.

ㅇ mehrfach vom südlichen Ussuri-Gebiet, Ostasien.

Bombus hortorum var. japonellus n. var. ‥

q. Wie B. diversus Sm., aber Segment 3-6 schwarz behaart, 1-2 gelbbraun, aber die Seiten von Segment 2 ebenfalls dicht schwarz behaart.

2 q von Yokohama (Japan), Andrea e leg.

Bombus hortorum var. raddefkaënsis n. var. 웅.

욱. Wie B. ussurensis Rad., aber Segment $4-6$ rein weifs behaart, Segment 1-2 wie der Thorax hellgelb behaart, mit dunkelgelber Scheibe, Segment 2 an den Seiten schwarz behaart, aber am Endrand weifs gefranst.

$\sigma^{7}+$ mehrfach von Ost-Sibirien (Raddefka) und von Irkutsk.

Von B. consobrinus Dlb. unterscheidet sich vorliegende Form durch die hellgelbe Behaarung und durch die weifs behaarte Unterseite, auch Thoraxseiten und Beine sind meist weifslich behaart.

Bombus pomorum var. krügeri n. var. + .

+. Wie B. pomorum, aber das Collare breit weifs behaart, Segment $1-3$ ganz schwarz, $4-6$ typisch rot behaart. Die Wangen etwas kürzer als beim typischen $B$. pomorum.

1 f von Salzburg, 1910 Friese leg. 


\section{$2 \mathrm{BHL}$ Biodiversity Heritage Library}

Friese, Heinrich. 1916. "Über einige neue Hummelformen (Bombus), besonders aus Asien. (Hym)." Deutsche entomologische Zeitschrift 1916(2), 107-110. https://doi.org/10.1002/mmnd.48019160205.

View This Item Online: https://www.biodiversitylibrary.org/item/103197

DOI: https://doi.org/10.1002/mmnd.48019160205

Permalink: https://www.biodiversitylibrary.org/partpdf/236239

\section{Holding Institution}

Harvard University, Museum of Comparative Zoology, Ernst Mayr Library

\section{Sponsored by}

Biodiversity Heritage Library

\section{Copyright \& Reuse}

Copyright Status: Public domain. The BHL considers that this work is no longer under copyright protection.

This document was created from content at the Biodiversity Heritage Library, the world's largest open access digital library for biodiversity literature and archives. Visit BHL at https://www.biodiversitylibrary.org. 